\title{
Charles Bonnet syndrome: visual loss and hallucinations
}

\section{M.L. Jackson MD, Joseph Ferencz MD PhD}

A 69-year-old man presented to his doctor with complaints of visual hallucinations that first occurred 6 months before presentation. He reported having hallucinations most evenings. He saw the faces of people, odd shapes, animals and a spider, which he had tried to kill. He also reported seeing people that he knew sitting in the armchair next to his night table.

The patient had no history of mood disorders or psychiatric or neurologic diseases. He had type 2 diabetes, hypertension, arthritis and coronary artery disease. His current medications included amlodipine, acetylsalicylic acid, clopidogrel bisulfate, glimepiride, hydrochlorothiazide, furosemide, lisinopril, metoprolol succinate, simvastatin and tramadol. He was also taking tamsulosin and dutasteride for benign prostatic hypertrophy and allopurinol for gout. None of his medications had been changed before the onset of the hallucinations.

The patient reported having an abrupt change in vision about 16 months before presentation, at which time he noted distortion in his vision. He received a diagnosis of exudative age-related macular degeneration and had 12 intraocular ranibizumab injections after diagnosis. On examination, he has clear cognition. His visual acuity was 20/70 in the right eye and 20/50 in the left eye.

\section{Discussion}

\section{Diagnosis}

A hallucination is "a sensory perception that has the compelling sense of reality of a true perception but that occurs without external stimulation of the relevant sensory organ."1 Visual hallucinations can include both formed images, such as people, and unformed images, such as flashes of light. In elderly patients, visual hallucinations may be caused by dementia, delirium, drug-induced states or primary psychiatric disorders.

Based on 3 characteristics (visual loss, clearly formed recurrent visual hallucinations, and insight into the unreal nature of the hallucinations), our patient received a diagnosis of Charles Bonnet syndrome.

\section{Loss of vision}

The reported prevalence of Charles Bonnet syndrome in people with visual impairment is $10 \%-38 \%$. This wide range is attributed to differences in definition, history-taking and patients' willingness to disclose the symptom because of concern that it implies mental incompetence. ${ }^{2}$ Historically, not all authors cited loss of vision as a component of the diagnosis. ${ }^{3}$ However, the association with visual loss is now widely

\section{Key points}

- Patients with loss of vision may experience vivid recurrent visual hallucinations, such as formed images of people or animals.

- Patients with Charles Bonnet syndrome have insight that the images are not real after this has been explained to them.

- Other diagnoses should be considered if the hallucinations are associated with neurologic symptoms, cognitive decline or lack of insight.

thought to be a required part of the diagnosis. Hallucinations as part of this syndrome can occur in patients of any age with any type or degree of visual loss.

\section{Hallucinations}

The hallucinations associated with Charles Bonnet syndrome can vary widely from simple, repeated coloured patterns to images such as double-decker buses coming into one's living room or people sitting on the couch.

There is no agreement among authors as to whether images of bright lights or diffuse colours are part of the spectrum of Charles Bonnet syndrome. However, the clear description of the shape or position of the image and recurrence of this symptom is suggestive of a hallucination that is part of Charles Bonnet syndrome.

Patients with this syndrome typically present a clear description of the hallucinations, which is characteristic of Charles Bonnet syndrome. Some patients report experiencing both auditory and visual hallucinations; however, the coexistence of visual and auditory components of Charles Bonnet syndrome is controversial. ${ }^{4}$

\section{Insight}

Although insight into the unreal nature of the images is required to make a diagnosis of Charles Bonnet syndrome, patients may not have full insight at the onset of the symptom. Patients may initially be confused about what they are experiencing and may act on their hallucinations. They may have to regularly reassure themselves that what they are seeing is not real, particularly if the image fits into the real setting.

From the Massachusetts Eye and Ear Infirmary (Jackson), Harvard Department of Ophthalmology, Boston, USA; and the Department of Psychiatry and Behavioural Neurosciences (Ferencz), McMaster University, Hamilton, Ont. 
To make a diagnosis of Charles Bonnet syndrome, however, the patient should appreciate that the images are not real after this has been fully explained to them. Lack of insight should lead to consideration of other diagnoses.

\section{Causes}

The specific cause of the hallucinations is unknown; however, they are most commonly attributed to deafferentation or lack of true visual input into the brain, which causes a release phenomenon similar to phantom limb symptoms after amputation. ${ }^{5}$ The deafferentation theory is supported by the results of a study that included 13 normally sighted patients who were blindfolded for 5 consecutive days. Of these patients, 10 reported hallucinations after an average of 1 day. ${ }^{6}$

\section{Differential diagnosis}

Ocular disease affects a disproportionate number of elderly patients. Charles Bonnet syndrome is, therefore, most common in this population. The differential diagnosis of hallucinations in an elderly patient includes psychiatric diseases with prominent psychotic symptoms (e.g., bipolar disorder) and neurologic diseases (e.g., Alzheimer disease, Parkinson disease).

Hallucinations have been described by up to $36 \%$ of patients with Parkinson disease. Although the hallucinations are often attributed to pharmacological treatments or Parkinson psychosis, a study that included 30 patients with untreated Parkinson disease found that $25 \%$ of the patients had hallucinations. These patients had insight and did not have cognitive impairment, suggesting that these hallucinations have characteristics in common with Charles Bonnet syndrome.

Hallucinations may also be prominent in delirium, drugintoxication states and drug-withdrawal states (particularly alcohol withdrawal) and may also occur as an adverse effect of medication. Hallucinations may also occur in the hypnagogic (before sleep) and hypnopompic periods (before wakefulness).

Patients with ocular disease and hallucinations who present with difficulty with mentation, poor insight or other neurologic signs or symptoms, as reported by the patient or the patient's family, should be referred for psychiatric or neurologic evaluation.

A history of clearly described recurrent visual hallucinations in a patient with known ocular disease who has insight after the unreal nature of the hallucinations is explained supports a diagnosis of hallucinations as part of Charles Bonnet syndrome.

\section{Treatment}

Some patients note improvement or resolution of the visual hallucinations over several years, but it is not clear which factors are linked to resolution. The natural history of Charles Bonnet syndrome has not been studied in a large series of patients. ${ }^{8}$

Most patients who experience hallucinations as part of Charles Bonnet syndrome are not bothered by the symptom and, hence, are not candidates for pharmacological treatment. However, some patients are disturbed by the hallucinations and may benefit from treatment of this unusual symptom. A few case reports indicate that Charles Bonnet syndrome has been successfully treated with medications including risperidone, cisapride, valproate, carbamazepine, clonazepam, selec-

\section{Who is Charles Bonnet?}

Charles Bonnet (1720-1793) was a Swiss naturalist and philosopher. In 1760, he described the vivid hallucinations experienced by his 87 year-old grandfather. He recognized that his grandfather was clear thinking and that the hallucinations were associated with vision loss.

He described his grandfather as: "A respectable man full of health, of ingenuousness, judgement, and memory, who completely alert and independently from all outside influences sees from time to time, in front of him, figures of men, of women, of birds, of carriages, of buildings... All of this appears to have a seat in that part of the brain involved with sight." ${ }^{16}$ The eponym using his name is a reminder of this association between hallucinations and vision loss.

tive serotonin reuptake inhibitors, gabapentin and olanzapine. ${ }^{9-15}$ The evidence for treatment remains limited.

Given the prevalence of Charles Bonnet syndrome in patients with known ocular disease, physicians may consider asking patients in this group about visual hallucinations. If a diagnosis of Charles Bonnet syndrome is made, patients should be counselled about the nature of these hallucinations. Recognizing a patient's report of visual hallucinations as a symptom of vision impairment can avoid misdiagnosis, unnecessary investigation and possible mismanagement.

Competing interests: None declared.

\section{REFERENCES}

1. American Psychiatric Association. Diagnostic and statistical manual of mental disorders. 4th ed. Arlington (VA): The Association; 1994.

2. Jackson ML, Bassett K, Nirmalan PV, et al. Contrast sensitivity and visual hallucinations in patients referred to a low vision rehabilitation clinic. $\mathrm{Br} J$ Ophthalmol 2007;91:296-8.

3. Menon GJ, Rahman I, Menon SJ, et al. Complex visual hallucinations in the isually impaired: the Charles Bonnet syndrome. Surv Ophthalmol 2003;48:58-72.

4. Hori H, Terao T, Nakamura J. Charles Bonnet syndrome with auditory hallucinations: a diagnostic dilemma. Psychopathology 2001;34:164-6.

5. Fernandez A, Lichtshein G, Vieweg V. The Charles Bonnet syndrome: a review. J Nerv Ment Dis 1997;185:195-200.

6. Merabet LB, Maguire D, Warde A, et al. Visual hallucinations during prolonged blindfolding in sighted subjects. J Neuroophthalmol 2004;24:109-13.

7. Biousse V, Skibell BC, Wattts R, et al. Ophthalmologic features of Parkinson's disease. Neurology 2004;62:177-80.

8. Holroyd S, Rabins PV. A three-year follow-up study of visual hallucinations in patients with macular degeneration. J Nerv Ment Dis 1996;184:188-9.

9. Howard R, Meehan O, Powell R, et al. Successful treatment of Charles Bonnet syndrome type visual hallucinosis with low dose risperidone. Int J Geriatr Psychiatry 1994;9:677-8.

10. Ranen NG, Pasternak RE, Rovern BW. Cisapride in the treatment of visual hallucinations caused by visual loss: the Charles Bonnet syndrome. Am J Geriatr Psychiatry 1999;7:264-6.

11. Hori H, Terao T, Shirashi Y, et al. Treatment of Charles Bonnet syndrome with valproate. Int Clin Psychopharmacol 2000;15:117-9.

12. Terao T. Effect of carbamazepine and clonazepam combinationon on Charles Bonnet syndrome: a case report. Hum Psychopharmacol Clin Exp 1998;13:451-3.

13. Lang UE, Stogowdki D, Schulze D, et al. Charles Bonnet syndrome: successful treatment of visual hallucinations due to vision loss with selective serotonin reuptake inhibitors. J Psychopharmacol 2007;21:553-7.

14. Holroyd S, Sabeen S. Successful treatment of hallucinations associated with sensory impairment using gabapentin. J Neuropsychiatry Clin Neurosci 2008;20:364-6.

15. Coletti Moja M, Milano E, Gasverde S, et al. Olanzapine therapy in hallucinatory vision related to Bonnet syndrome. Neurol Sci 2005;26:168-70.

16. Hedges TR Jr. Charles Bonnet, his life, and his syndrome. Surv Ophthalmol 2007;52:111-4. 